\section{Beating organs-on-chip as technological platforms in drug screening: Advanced in vitro models of human physiology and pathology}

\author{
Paola Occhetta, ${ }^{1}$ Roberta Visone, ${ }^{1}$ \\ Stefano Piazza, ${ }^{1}$ Ferran Lozano, 1,2 \\ Marco Rasponi2 \\ 1BiomimX S.r.l., Milano, Italy; \\ 2Politecnico di Milano, Milano, Italy
}

\begin{abstract}
Taking advantages of $u B e a t^{\circledR}$ technology, 3D beating Organs-on-Chip integrates the native complexity of human mechanical microenvironment into clinically relevant in vitro models of human organs and diseases.
\end{abstract}

\section{Introduction}

Organs-on-Chip (OoC) have recently emerged as innovative in vitro tools holding the potential to improve prediction over human drug responses. However, bringing into $\mathrm{OoC}$ models the entire complexity of native human tissue microenvironmental cues is still not trivial. Here we present new 3D beating $\mathrm{OoC}$, advanced miniaturized platforms integrating for the first time the native-like $3 \mathrm{D}$ mechanical microenvironment with an unprecedented level of precision. This is achieved through uBeat ${ }^{\circledR}$, an innovative technology ${ }^{1}$ that allows to modulate mechanical deformation exerted on 3D microtissues in a controlled fashion. As case studies, two $u$ Beat ${ }^{\circledR}$-based models are presented: i) uHeart, a beating heart-on-chip integrating real-time electrophysiological measurements, and ii) uKnee, the first in vitro model of human osteoarthritic (OA) cartilage-on-chip.

\section{Materials and Methods}

$\mathrm{uBeat}^{\circledR}$ technology ${ }^{1}$ provides miniaturized cells culture in 3D with highly controlled and tunable mechanical stimulation patterns. Relying on specific geometrical features that modulate the mechanical deformation exerted on 3D microtissues, uBeat $^{\circledR}$ allows achieving either a uniaxial strain or a confined compression, as further detailed for the case studies.

In the uHeart model, uBeat ${ }^{\circledR}$ is exploited to provide $3 \mathrm{D}$ human cardiac microtis- sues with a physiological cyclic uniaxial strain (i.e. $10 \%, 1 \mathrm{~Hz}$ ). Cardiac microtissues are generated using cardiomyocytes from human induced pluripotent stem cells (hiPSC-CMs) and human dermal fibroblast, embedded in fibrin (total concentration of $100-125 \cdot 10^{6}$ cells $/ \mathrm{ml}$, ratio $3: 1$ ) and cultured within uHeart for 7 days. Electrical activity is monitored on-line to track microtissue development and to characterized beating parameters (e.g., beating period, FP spike amplitude, FP duration). FP morphology changes can be evaluated upon administration of drugs affecting cardiac electrical activity (e.g., Sotalol and Verapamil) or not (e.g., Aspirin). In the uKnee model, uBeat ${ }^{\circledR}$ is exploited to provide 3D miniaturized cartilage-like constructs (namely Cartilage-onChip, CoC) with Hyper-Physiological (HP) compression (i.e. $30 \%, 1 \mathrm{~Hz}$ ) with the aim to elicit OA pathogenesis in vitro. Upon two weeks of culture of healthy cartilage microconstructs from human articular chondrocytes embedded in a poly(ethylene-glycol)based (PEG) hydrogel, HP compression is applied for 7 additional days. The induction of OA-like traits is verified via immunofluorescence and qPCR. Reversal of OA traits upon administration of anti-inflammatory and anti-degrading drugs or medical devices (MD) can be assessed in uKnee model.

\section{Results}

Upon seven days of mechanical training resembling the heartbeat, ${ }^{2}$ human cardiac cells cultured within uHeart developed in synchronously beating and functional cardiac microtissues. This was demonstrated both via immunofluorescence staining for Connexin43 (Cx43) and Myosin Light Chain 2 (MLC2) and by electrophysiological studies conducted directly on-chip, which enable the continuous monitoring of constructs' electrical activity (Figure 1a). ${ }^{3}$ Cardiac microtissues spontaneously beat as a syncytium with a RR of $1.7 \pm 0.45 \mathrm{~s}$, a FP duration of $0.6 \pm 0.2 \mathrm{~s}$ and a spike amplitude of $590 \pm 440 \mu \mathrm{V}$. Drug screening results evidenced that Aspirin did not affect the repolarization time, while Sotalol prolonged and Verapamil shorten the FP duration of human microtissues (Figure 1b).

Human articular chondrocytes statically cultured in the platform within a PEG based gel for 14 days formed a mature articular cartilage on chip (CoC), expressing genes characterizing human articular cartilage and interzone (e.g. PRG4, GDF5, ATX) and producing articular cartilage matrix (i.e. aggrecan and Collagen type-II).

A $30 \%$ confined HP compression reca-
Correspondence:Paola Occhetta, BiomimX S.r.l., Milano, Italy.

E-mail: paola.occhetta@biomimx.com

Key words: Organs on chip; mechanical stimulation; disease modeling; drug screening.

Acknowledgments: Device manufacturing was partially performed at PolifAB (Politecnico di Milano). This project has received funding from the European Union's Horizon 2020 research and innovation programme under the Marie Skłodowska-Curie grant agreement No 860715

Disclosures: P.O., R.V. and M.R. share equities in BiomimX.

Conference presentation: This paper was presented at the Third Centro 3R Annual Meeting - L'era delle 3R: modelli in silico, in vitro e in vivo per promuovere la ricerca traslazionale 30 September - 1 October 2021, Evento online organizzato dal Politecnico di Torino.

Received for publication: 9 July 2021.

Accepted for publication: 7 September 2021.

This work is licensed under a Creative Commons Attribution NonCommercial 4.0 License (CC BY-NC 4.0).

C Copyright: the Author(s), 2021

Licensee PAGEPress, Italy

Biomedical Science and Engineering 2021; 4(s1):141 doi:10.4081/bse.2021.14

pitulating the mechanical factors involved in OA pathogenesis was sufficient to induce OA traits in the $\mathrm{CoC}^{4}$, accounting for i) shift of homeostasis towards catabolism and triggering of inflammation (IL6 and IL8 upregulation, MMP13 production), ii) trigger of hypertrophy (COL10A1 and IHH upregulation) and, iii) acquisition of a gene profile correlating with clinical OA evidences (decrease expression of FRZB and GREMI, Figure 2a). Finally, during additional 3 days of cyclic compression, available anti OA drugs were tested and the effect on $M M P 13$ and IL8 modulation was attested, probing the platform value as a disease modifying OA drugs screening tool (Figure 2b).

\section{Discussion and Conclusions}

Integration of 3D mechanical microenvironment resulted in OoC models with enhanced functionality and resemblance to pathological states. Both models presented as case studies were successfully exploited for drug screening purposes, by testing the effect of both well-known drugs and com- 
pounds under development and demonstrating the potentiality of $3 \mathrm{D}$ beating $\mathrm{OoC}$ as drug/MD screening tool. uBeat ${ }^{\circledR}$ is nevertheless highly versatile and applicable to any organ/disease in which mechanical stimulation exerts a pathophysiological state. uBeat ${ }^{\circledR}$ represents a powerful preclinical tool for efficient in vitro drug screening/disease modelling, towards a future of precision medicine.
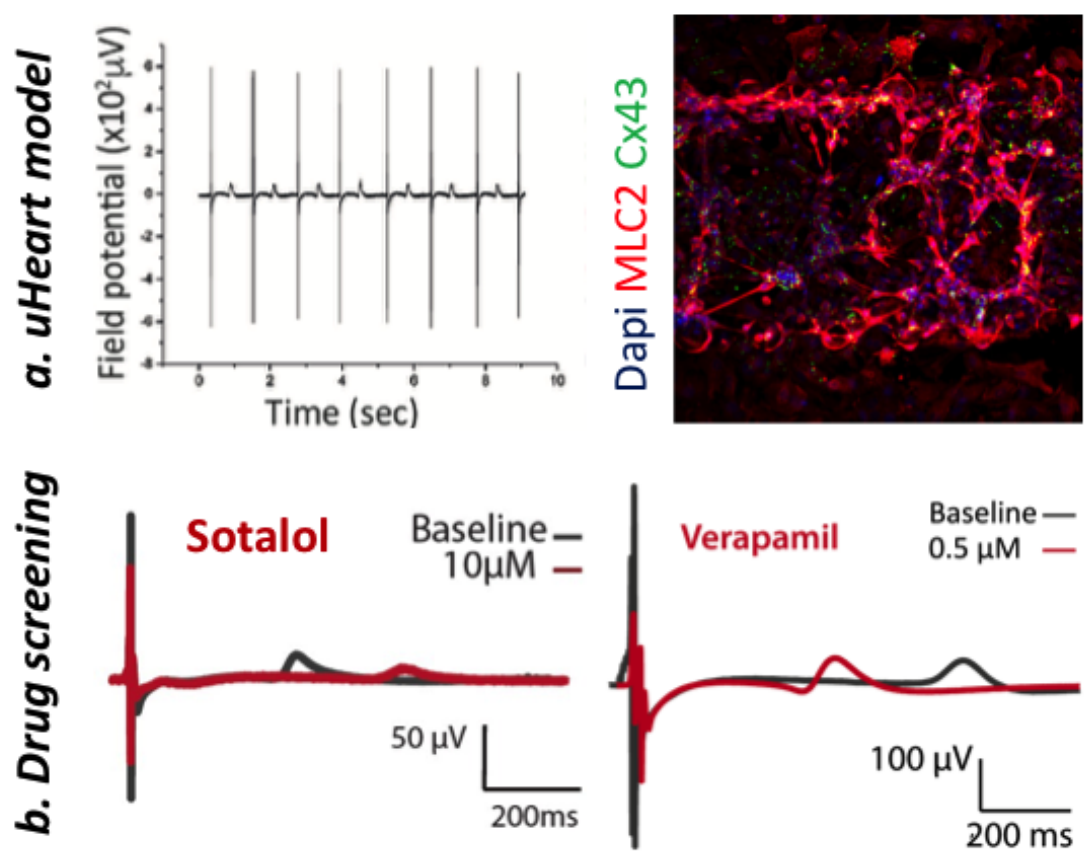

\section{References}

1. Redaelli A, Rasponi M, Occhetta P. Microfluidic device and relative method for the generation and/or culture and/or maturation of three- dimensional cell and/or tissue constructs. European Patent Office EP3289065B1, 2015.

2. Marsano A, Conficconi C, Lemme M, et al. Beating heart on a chip: a novel microfluidic platform to generate functional 3D cardiac microtissues. Lab Chip 2016;16:599-610.

3. Visone R, Ugolini GS, Cruz-Moreira D, et al. Micro-electrode channel guide $(\mu \mathrm{ECG})$ technology: an online method for continuous electrical recording in a human beating heart-on-chip. Biofabrication 2021;13:035026.

4. Occhetta P, Mainardi A, Votta E, et al. Hyperphysiological compression of articular cartilage induces an osteoarthritic phenotype in a cartilageon-a-chip model. Nat Biomed Eng 2019;3:545-57.

Figure 1. Field potential directly measured from uHeart model and immunofluorescence of functional cardiac microtissues upon uBeat ${ }^{\circledR}$ mechanical training (a). Effect of Sotalol and Verapamil on field potential (b).

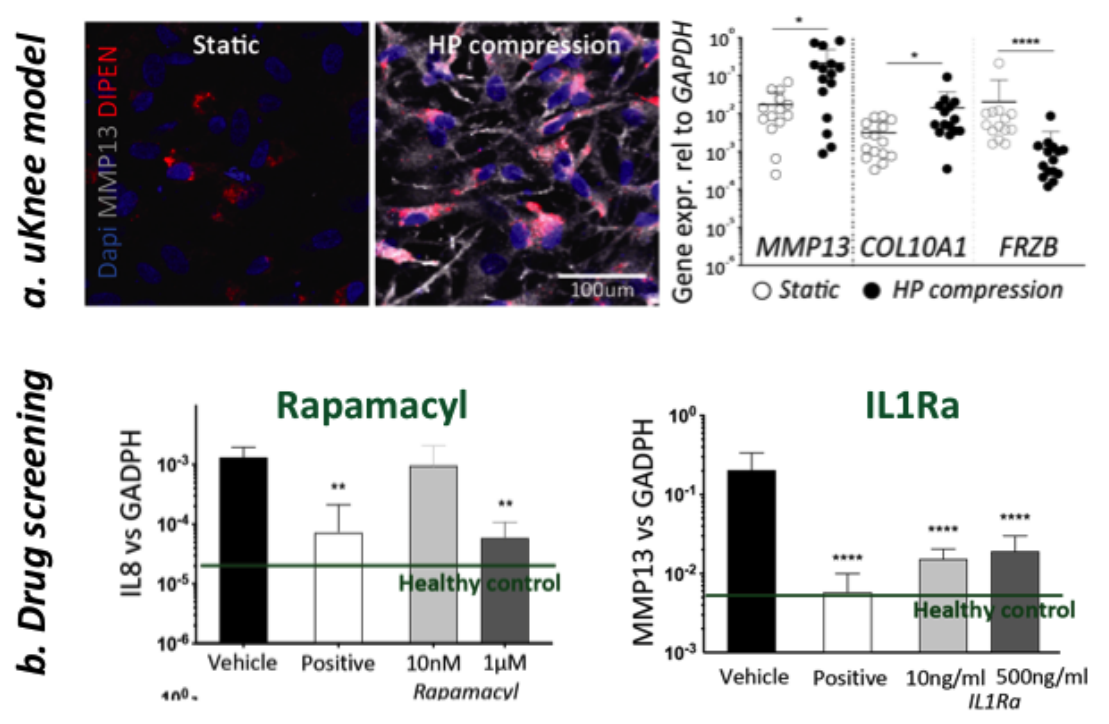

Figure 2. uBeat ${ }^{\circledR}$-based induction of OA traits in CoC (a). Effect of Rapamacyl and IL1Ra on reduction of inflammation and matrix degradation (b). 